\title{
Analysis of the molecular mechanism of Evodia rutaecarpa fruit in the treatment of nasopharyngeal carcinoma using network pharmacology and molecular docking
}

\section{Runshi Xu}

Hunan University of Chinese Medicine, Hanpu Science and Education Park

\section{Ximing Yang}

Hunan University of Chinese Medicine, Hanpu Science and Education Park

\section{Yangyang Tao}

Hunan University of Chinese Medicine, Hanpu Science and Education Park

\section{Wang Luo}

Hunan University of Chinese Medicine, Hanpu Science and Education Park

\section{Yu Xiong}

Hunan University of Chinese Medicine, Hanpu Science and Education Park

\section{Lan He}

First Affiliated Hospital of Hunan University of Traditional Chinese Medicine

\section{FangLiang Zhou}

Hunan University of Chinese Medicine, Hanpu Science and Education Park

Yingchun He ( $\nabla$ heyingchun@hnucm.edu.cn )

Hunan University of Chinese Medicine, Hanpu Science and Education Park

\section{Research Article}

Keywords: nasopharyngeal carcinoma, Evodia rutaecarpa, interaction network analysis, disease-related gene mining, target-gene prediction, protein-protein interaction network

Posted Date: March 14th, 2022

DOI: https://doi.org/10.21203/rs.3.rs-1266309/v3

License: (c) (1) This work is licensed under a Creative Commons Attribution 4.0 International License. Read Full License 
Version of Record: A version of this preprint was published at Journal of Healthcare Engineering on April 13th, 2022. See the published version at https://doi.org/10.1155/2022/6277139. 


\section{Abstract}

Background: Nasopharyngeal carcinoma (NPC), a neoplasm of the head and neck, has high incidence and mortality rates in East and Southeast Asia. Evodia rutaecarpa is a tree that is native to Korea and China, and its fruit (hereafter referred to as Evodia) exhibits remarkable antitumor properties. However, little is known about its mechanism of action in NPC. In this study, we employed network pharmacology to identify targets of active Evodia compounds in nasopharyngeal carcinoma and generate an interaction network.

Methods: The active ingredients of Evodia and targets in NPC were obtained from multiple databases, and an interaction network was constructed via the Cytoscape and STRING databases. The key biological processes and signaling pathways were predicted using Gene Ontology and Kyoto Encyclopedia of Genes and Genomes pathway enrichment analyses. Molecular docking technology was used to identify the affinity and activity of target genes, and The Cancer Genome Atlas and Human Protein Atlas databases were used to analyze differential expression. Cell Counting Kit (CCK)-8 and Annexin V-fluorescein isothiocyanate (FITC)/propidium iodide (PI) dual-fluorescence staining were used for experimental verification.

Results: Active Evodia compounds included quercetin, isorhamnetin, and evodiamine, and important NPC targets included MAPK14, AKT1, RELA, MAPK1, JUN, and p53, which were enriched in lipid and atherosclerosis signaling pathways. Additionally, we verified the high affinity and activity of the active compounds through molecular docking, and the target proteins were verified using immunohistochemistry and differential expression analyses. Furthermore, CCK-8 assays and Annexin VFITC/PI dual-fluorescence staining showed that isorhamnetin inhibited the proliferation of NPC cells and induced apoptosis.

Conclusion: Our results identified the molecular mechanisms of Evodia and demonstrated its ability to alter the proliferation and apoptosis of NPC cells through multiple targets and pathways, thereby providing evidence for clinical application of Evodia.

\section{Introduction}

Nasopharyngeal carcinoma (NPC) is an Epstein-Barr virus (EBV)-associated epithelial head and neck neoplasm, with the highest incidence and mortality rates occurring in East and Southeast Asia and a 5year survival rate of only approximately $50 \%$ for patients with advanced disease [1,2]. NPC is highly malignant, has a high recurrence rate, is prone to distant metastasis, and occurs as an advanced disease in more than $50 \%$ of patients at diagnosis [3]. NPC is a major public health problem in many countries, particularly in Southeast Asia and North Africa, and its etiology is considered to be related to various risk factors, such as a family history of the disease, diet containing high-risk ingredients (such as large amounts of nitrosamine compounds and volatile nitrosamines), and previous EBV infection [4,5]. Comprehensive treatment with conventional conformal radiotherapy and cisplatin-based adjuvant 
chemotherapy is the preferred regimen for treating NPC [6]; however, disease recurrence and distant metastasis are the main factors associated with treatment failure. Such a treatment regimen can also result in concurrent and severe adverse reactions; therefore, the development of a new model for treating NPC is critical.

The use of traditional Chinese medicine (TCM) to treat NPC is drawing increasing attention. TCM has fewer side effects and adverse reactions than Western chemotherapeutic drugs and presents a lower risk of patients developing drug resistance. Evodia (Tetradium ruticarpum (A.Juss.) T.G.Hartley [Rutaceae]) is the dry and nearly mature fruit of rutaceous plants [Evodia rutaecarpa (Juss.) Benth. var. Officinalis (Dode) Huang or Evodia rutaecarpa (Juss.) Benth. var. bodinieri (Dode) Huang]. Recent studies have shown that the main Evodia compounds are alkaloids, bitterin, volatile oil, and flavonoids, among which quercetin, evodiamine, and isorhamnetin are the main active compounds [7-9]. A pharmacological study indicated that Evodia has analgesic, anti-inflammatory, anti-ulcer, and antitumor effects in addition to other protective effects, such as those acting on the cardiovascular system [10]. Moreover, Evodia is an important compatible medicinal material used in a variety of prescriptions in TCM. However, its chemical composition is complex and includes many biologically active compounds that interact with multiple NPC-related therapeutic targets.

With the rapid growth and diversification of biological and drug data, it is necessary to adopt more extensive analytical techniques in systems biology. Network pharmacology methods based on systems biology and multidirectional pharmacology may be helpful to understand the interactions of various active components and solve their mechanisms of action. Several synergistic compounds in TCM prescriptions or monomers have been screened in a high-throughput manner, and the network regulation and rules have been elucidated.

Developing an Evodia-based treatment model that affects multiple signaling pathways based on its variety of active compounds could provide new strategies for treating diseases with complex pathophysiologies and multiple targets. Network pharmacology methods based on systems biology and polypharmacology can help elucidate interactions between various active compounds. The complete method uses a "disease-target-drug" interaction network to identify connections between the active compounds of a drug and the disease target and attempts to clarify the synergy between the compounds and their potential mechanisms. Therefore, this method could clarify the mechanism of action of Evodia for NPC.

Current research on Evodia mainly focuses on its application for the treatment of cardiovascular diseases and other nontumor diseases; however, its applicability to tumors, particularly those related to NPC, requires urgent attention. Therefore, in this study, we used network pharmacology and molecular-docking studies to predict the therapeutic targets of Evodia and its possible mechanisms of action in treating NPC. We subsequently verified the results using transcriptomic analysis and experiments. These results can provide a reference for subsequent research and promote new strategies involving the use of Evodia as targeted therapy for NPC. 


\section{Material And Methods}

\subsection{Screening of active pharmaceutical compounds}

We used the TCM Systems Pharmacology (TCMSP) Database and Analysis Platform to retrieve the chemical composition of Evodia [11]. To obtain the biologically active Evodia compounds, the search results were screened using oral bioavailability $(\mathrm{OB} ; \geq 30 \%)$ and drug-likeness ( $\mathrm{DL} ; \geq 0.18)$ as the restrictive conditions [12]. Potential Evodia targets were then collected through the TCMSP platform and standardized using the UniProt database [13].

\subsection{Disease-related gene mining}

To obtain NPC targets, we used "nasopharyngeal carcinoma" as a keyword for searches of the following five databases: GeneCard, OMIM, PharmGkb, the therapeutic targets database, and Drugbank. We merged the search results, deleted duplicate items, and obtained all NPC-related target genes.

\subsection{Predicting the therapeutic target gene set}

We matched the target-prediction results of the effective active Evodia compounds with the search results of NPC-related target genes and then used the Venn R package to compile a Venn diagram comprising targets of the effective active Evodia compounds and NPC target genes. We extracted cross-target Evodia genes and NPC targets as a common gene set to determine potential Evodia compounds for the treatment of NPC and then analyzed the results.

\subsection{Constructing a network of active compounds and associated disease targets}

The screening results for the active Evodia compounds were imported into Cytoscape along with the NPC target-screening results [14] to construct a network of active compounds and NPC targets. We then used Network Analyzer to analyze the topological properties of the network, including the degree, betweenness centrality, and closeness centrality.

The nodes in the network are represented by a protein target or active compound, and the edges represent the interaction between these biomolecules. A node is considered to have a greater impact when it has more direct connections with other nodes. The centrality between nodes is a measurement of the shortest path between them, and closeness centrality is defined as the reciprocal of the average value of the shortest distances between a node and all other nodes in the network. The larger the value, the greater the centrality of the node, which also relates to the speed at which a signal is transmitted from one node to another $[15,16]$. The node degree indicates the number of nodes connected in the network. A node with 
high betweenness centrality (BC) and closeness centrality (CC) values plays a very important role in the network.

\subsection{Construction of a protein-protein interaction (PPI) network and core gene screening}

A PPI is formed when two or more protein molecules form a complex through noncovalent bonds. PPIs and the resulting networks are crucial in most biological functions and processes [17]. The STRING database contains known and predicted PPIs, with each PPI annotated with one or more "scores" that are not indicative of the strength or specificity of the interaction but which represent an indicator of confidence and, thus, how likely STRING is to judge an interaction to be true based on the available evidence. All scores are ranked from 0 to 1, with 1 representing the highest possible confidence. A score of 0.5 indicates that roughly every second interaction is erroneous (i.e., a false positive). Based on this, the common gene targets of Evodia and NPC were imported into the STRING database. Using the Homo sapiens species, a confidence level of 0.7 , and hidden disconnected nodes in the network, we performed a PPI network analysis and downloaded the results in TSV format.

The file was then imported into Cytoscape to conduct topology analysis, and CytoNCA was employed to calculate the six parameters used to evaluate the topological properties of the nodes within the interaction network [18]. These included the following: "degree centrality" (DC), BC, CC, "eigenvector centrality" (EC), "network centrality" (NC), and "local average connectivity" (LAC). These six parameters were used to indicate the nature of the nodes and determine their significance in the network. A node with high DC, BC, CC, EC, NC, and LAC values was considered to play an important role in the network. According to the results of the topological properties of the PPI network, targets above the median were selected as core targets, and two screen tests were conducted. Therefore, the core targets in the network were obtained.

\subsection{Gene Ontology (GO) biological functional analysis and Kyoto Encyclopedia of Genes and Genomes (KEGG) pathway enrichment analysis}

GO and KEGG pathway enrichment analyses were conducted to analyze the target genes. An absolute value of the logarithmic fold change (>0.5) was the criterion for judging the significance of differentially expressed genes $[19,20]$. The GO enrichment analysis provides a framework to describe the function of gene products in all organisms and to identify the biological characteristics or transcriptome data of high-throughput genomes. It uses three terms: molecular function, biological process, and cellular component. The KEGG database provides mRNA-related biological pathways enriched according to a given set of data. These analyses were conducted using the clusterProfiler R package [21], and the ggplot2 R package was used to display the results. 


\subsection{Molecular docking to identify interactions between drug and target compounds}

Target protein structures were obtained from the Research Collaboratory for Structural Bioinformatics (RCSB) Protein Data Bank (PDB), and the two-dimensional structures of the active Evodia compounds were obtained from the PubChem database [22]. ChemBio Draw 3D (PerkinElmer, Billerica, MA, USA) and Autodock (https://autodock.scripps.edu/) were used to optimize the structures of key compounds and targets, which were used to determine three-dimensional (3D) chemical structures and perform energy minimization and format conversion [23]. PyMol was used to evaluate the proteins, remove ligands and water molecules, and perform structural adjustment. Docking was carried out using R software and Autodock VINA. The molecule with the lowest binding energy in the docking conformation was selected, and the binding effect was observed via matching of the original ligand and the interaction between the molecules.

\subsection{Differential expression analyses}

Based on the high specificity of antibody binding to antigen, immunohistochemistry can reveal the relative distribution and abundance of proteins. Immunohistochemical data were obtained from the Human Protein Atlas (HPA), and the expression of target genes in normal and cancer tissues was compared [24]. Transcriptome data of cancer-related genes were obtained from The Cancer Genome Atlas (TCGA), including data related to 44 normal samples and 502 cancer samples. Differential expression analysis was conducted to verify the differences between the expression of target genes in cancer and cancer-adjacent samples. Additionally, transcription data of cancer and cancer-adjacent tissues from the same patient were extracted and paired to conduct differential expression analysis.

\subsection{In vitro experimental evaluation}

Different mass concentrations $\left(5,10,20,40\right.$, and $\left.80 \mu \mathrm{mol} \cdot \mathrm{L}^{-1}\right)$ of isorhamnetin and a positive control (cisplatin, $4 \mu \mathrm{g} \cdot \mathrm{mL}^{-1}$ ) were used to treat NPC CNE-2 cells. Cell Counting Kit (CCK)-8 assays were used to detect the proliferation of CNE-2 cells, and Annexin V-fluorescein isothiocyanate (FITC)/propidium iodide (PI) dual-fluorescence staining was employed to detect the apoptosis rate [25].

\subsection{Statistical analysis}

R software (v.3.6.1) was used to conduct statistical analyses. Unpaired $t$-tests were used to compare two groups of normally distributed variables, and the statistical significance of the non-normally distributed variables was estimated using the Mann-Whitney Utest (Wilcoxon rank-sum test). The experimental verification results were processed using SPSS software (v.26.0; IBM Corp., Armonk, NY, USA), and data were visualized using GraphPad Prism software (v.8.0; GraphPad Software, La Jolla, CA, USA). The false 
discovery rate (FDR) method was used to adjust the $p$ value for multiple comparisons, and statistical significance was set to $P<0.05$ (FDR $<0.05)$.

\section{Results}

\subsection{Research process}

Figure 1 shows a flowchart describing the process used to determine the effective target genes of Evodia for treating NPC and the experiments conducted to verify the results. The data sources used in this process and the websites visited for data analyses are provided in Table S1.

\subsection{Screening of active Evodia compounds}

In the TCMSP database, 30 active Evodia compounds were selected via screening, where OB greater than $30 \%$ and DL greater than 0.18 were the restrictive conditions (Table S2). Together with the compound retrieval results, we identified 24 active compounds for subsequent experiments (including indoles, quinolone alkaloids, physalin, and volatile oils) where certain compounds, such as quercetin, have been confirmed to inhibit the growth of tumor cells [26].

Additionally, 495 Evodia targets were obtained from the TCMSP database. These genes were integrated through the UniProt database, and invalid and duplicate targets were deleted. In addition, 170 target genes associated with the effective active compounds were acquired (Table S3).

\subsection{Construction of a target gene set for NPC}

By integrating information from the GeneCard, OMIM, PharmGkb, TTD, and Drugbank databases, we obtained 8,953 gene targets for NPC (Figure 2A). We mapped the 170 target genes associated with the effective active Evodia compounds to the 8,953 target genes for NPC, identifying 153 composite target genes (Figure 2B and Table S4).

\subsection{Identification of active compounds in the Evodia-NPC- target interaction network}

We used Cytoscape software to draw a diagram of the active compounds in the Evodia-NPC-target interaction network. The docking results for the 24 active Evodia compounds and 153 NPC targets (Figure $2 \mathrm{C}$ and Table S5) resulted in a network of 177 nodes and 269 edges. The complex interactions between nodes indicated that individual target genes of NPC were associated with one or more active Evodia compounds, with multiple targets also corresponding to the same active compounds, suggesting that Evodia contains multiple compounds for multiple targets potentially related to NPC treatment. We 
identified quercetin, isorhamnetin, $\beta$-sitosterol, evodiamine, goshuyuamide, and rutaecarpine as the primary Evodia compounds associated with potential NPC targets.

\subsection{Construction of a target PPI network}

We then used STRING to process the NPC targets of Evodia compounds to construct a PPI network, resulting in a network of 127 nodes and 1,006 edges (Figure 3A). Scoring of the network in Cytoscape according to $B C, C C, D C, E C, N C$, and $L A C$ parameters (Figure $3 B$ ) revealed that the thresholds for the first round of screening were DC greater than 12 , EC greater than 0.042378277 , LAC greater than $4, B C$ greater than 58.28259727 , CC greater than 0.352941176 , and NC greater than 4.8 , with the results showing 33 nodes and 363 edges (Figure $3 C$ ). We then scored these nodes a second time, and the resulting thresholds for the second round of screening were DC greater than 20, EC greater than 0.148225084 , LAC greater than $9.142857143, \mathrm{BC}$ greater than $14.7986531, \mathrm{CC}$ greater than 0.581818182 , and NC greater than 10.29488406. These results revealed 13 nodes and 120 edges (Figure 3D and Table S6), including retinoblastoma 1 (RB1), c-Jun N-terminal kinase (JUN), mitogen-activated protein kinase (MAPK) 14, cyclin D1, AKT1, heat shock protein 90 alpha family class A member 1, estrogen receptor 1, MAPK1, MYC, hypoxia-inducible factor 1A, REL-associated protein (RELA), FOS, and p53. Therefore, these proteins were identified as the potential main therapeutic targets for treating NPC.

\subsection{Biological functional $\mathrm{GO}$ analysis and core pathway screening for Evodia in the treatment of NPC}

We used the Bioconductor R software package to conduct GO enrichment analysis of key targets. The screening was performed using a threshold of $P<0.05(\mathrm{Q}<0.05)$, resulting in the identification of 2118, 56 , and $181 \mathrm{GO}$ targets related to biological processes, cellular components, and molecular functions, respectively. The biological processes included responses to drugs, generation of reactive oxygen species (ROS), cellular response to chemical stress, response to lipopolysaccharides, and response to molecules of a bacterial origin. The top $20 \mathrm{GO}$ terms are displayed in Figures $4 \mathrm{~A}$ and $\mathrm{B}$ and in Table S7.

KEGG enrichment analysis of the 153 Evodia targets showed that they were enriched in 177 signaling pathways, including those related to hepatitis B, fluid shear stress and atherosclerosis, chemical carcinogenesis-receptor activation, and lipid and atherosclerosis, each of which play important roles in NPC development and progression. We displayed the top 20 KEGG enrichment results in Figures 4C and D and in Table S8. Notably, pathways associated with lipids and atherosclerosis were the most highly enriched signaling pathways (Figure 5). The GO and KEGG results showed that Evodia compounds potentially act on multiple NPC-specific pathways. 


\subsection{Molecular docking studies to evaluate interactions between active Evodia compounds and NPC targets}

Based on the KEGG enrichment analysis, the top six target proteins in the PPI network and their corresponding active compounds were selected for molecular docking. Data for the following proteins were downloaded from the PDB: MAPK14 (PDB ID: 2RG6), AKT1 (PDB ID: 7NH5), RELA (PDB ID: $5 U R N)$, JUN (PDB ID: 5T01), MAPK1 (PDB ID: 7NR9), and p53 (PDB ID: TBWN). Docking of the 3D structures with the respective compounds was visualized using PyMol, with lower binding energy designating a highaffinity interaction (Figure 6). The binding energy values of most Evodia compounds were below -1 $\mathrm{kcal} \bullet \mathrm{mol}^{-1}$, indicating that they possessed good binding activity. The results identified hydrogen bonding and $\pi-\pi$-stacking interactions, which suggested potential favorable interactions between the compounds and the NPC targets.

\subsection{Differential expression of target genes in NPC}

To verify the potential of the identified proteins as therapeutic targets, we analyzed the differential expression of proteins in NPC tissues and cancer-adjacent tissues. Immunohistochemical results from the HPA database showed that JUN expression in cancer tissues was higher than that in normal nasopharyngeal tissues, whereas antibody staining levels of MAPK14, AKT1, and MAPK1 in cancer tissues were lower than those in adjacent tissues. However, we observed no significant differences in RELA and p53 expression between tumor and normal nasopharyngeal tissues (Figure 7A).

We used TCGA database to expand the sample size for verification and obtained more accurate results. The box plot of the differential expression analysis (Figure 7B) showed that the expression of MAPK14, MAPK1, AKT1, and RELA was significantly higher in cancer tissues than in normal tissues. Furthermore, the paired analysis identified significantly higher MAPK1, AKT1, and RELA expression in cancer tissues than in cancer-adjacent tissues from the same patient (Figure 7C).

\subsection{Experimental verification}

Based on previous reports and pre-experimental results, we selected isorhamnetin for experimental verification of its influence on the biological processes of NPC cell proliferation and apoptosis. CCK-8 assays showed that treatment of CNE-2 cells with isorhamnetin $\left(5,10,20,40\right.$, and $\left.80 \mu \mathrm{mol} \cdot \mathrm{L}^{-1}\right) \mathrm{for} 24,36$, and $48 \mathrm{~h}$ significantly inhibited cell proliferation compared with cisplatin treatment $\left(4 \mu \mathrm{g} \cdot \mathrm{mL}^{-1} ; P<0.05\right.$; Figure 8A). Additionally, Annexin V-FITC/PI dual-fluorescence staining showed that isorhamnetin increased the apoptosis rate of NPC cells at $48 \mathrm{~h}$ (Figure 8B). These findings indicated that isorhamnetin induced NPC cell apoptosis.

\section{Discussion}


Given the increase in the prevalence of individualized diagnosis and treatment of disease, NPC morbidity and mortality have decreased to varying degrees in recent decades [27]. Preventing adverse reactions caused by individualized chemotherapy and intensity-modulated radiotherapy and increasing the clinical benefits to patients can positively impact the clinical success rate of NPC treatment. Numerous studies on NPC prevention and treatment have been conducted to identify new and effective treatment strategies that are less toxic and have fewer adverse effects than current regimens. In this respect, providing an individualized diagnosis and treatment regimen supplemented with natural products and their derivatives would be beneficial for treating NPC. TCM provides a more holistic approach to the diagnosis and treatment of tumors than Western medicine; the main pathogenesis of NPC is believed to be a deficiency of righteous qi, an internal accumulation of heat toxin, and intermingled phlegm and blood stasis. Of these, qi-deficiency-induced toxin contamination is regarded as the most important cause of NPC.

Evodia is recorded in ancient medicine books and has a long history of medicinal use. Modern pharmacological studies have shown that Evodia has analgesic [28], anti-inflammatory [29], antibacterial [30], antivomiting, antidiarrheal, and antitumor [31] properties, and biochemical analyses have demonstrated that Evodia mainly contains the following compounds: alkaloids, bitterin, volatile oil, and flavonoids. Of these, the most effective active compounds are reportedly quercetin, isorhamnetin, $\beta$ sitosterol, evodiamine, goshuyuamide, and rutaecarpine.

Previous studies have suggested that several active Evodia compounds exert cytotoxic effects on human cancer cell lines. Specifically, evodiamine has inhibitory effects on A549 human lung cancer cells [32], and limonin in Evodia induces apoptosis in ovarian cancer cells by activating the p53 signaling pathway [33]. Additionally, evodiamine plays an important role in activating c-Jun $\mathrm{N}$-terminal kinase (JNK) and protein kinase R-like endoplasmic reticulum kinase (PERK) in human ovarian cancer cells to induce apoptosis [34] and inhibits the proliferation of hepatocarcinoma cells through a WW domain containing oxidoreductase-dependent pathway to induce anticancer activity [35]. Moreover, evodiamine may induce bladder cancer cell apoptosis through mammalian target of rapamycin (mTOR)/S6 kinase 1mediated myeloid cell leukemia-1 downregulation and enhance tumor necrosis factor-related apoptosisinducing ligand-induced apoptosis [36]. These findings suggest the potential efficacy of Evodia in antitumor treatment.

However, Evodia displays a certain degree of hepatotoxicity, and adverse events have been reported in clinical practice. Therefore, exploring the specific Evodia compounds effective for tumor treatment, as well as their respective mechanisms of action, will help develop and promote their safe future use. Molecular docking is a computer analysis technology based on structures and ligands and is widely used to elucidate how compounds interact with their molecular targets and drug discovery and development. We searched for therapeutic compounds by reverse screening the ligands of the protein structure library and evaluating their binding affinity. This necessitates research on possible NPC targets of Evodia compounds. 
In this study, 24 active Evodia compounds and 153 NPC targets were used to construct a target interaction network, and the results showed that most Evodia compounds affected multiple NPC targets. Specifically, quercetin, isorhamnetin, evodiamine, and $\beta$-sitosterol interacted with 121, 26, 26, and 25 targets, respectively, suggesting that they may act as pleiotropic compounds. Although each compound showed interactions with multiple targets, many of these target-compound interactions overlapped, suggesting a possible synergistic effect between the compounds.

Quercetin is found in many fruits and vegetables, and many studies have investigated the molecular mechanisms of quercetin in NPC treatment. For example, a previous report confirmed that quercetin exerts anticancer effects on a variety of tumor cells by regulating the phosphatidylinositol 3-kinase (PI3K)/Akt/mTOR, Wnt/ $\beta$-catenin, and MAPK/extracellular signal-regulated kinase $1 / 2$ signaling pathways [26]. Additionally, quercetin inhibits angiogenesis in NPC tumors [37] and induces growth inhibition and apoptosis of NPC cells $[38,39]$. Furthermore, quercetin is used in cisplatin combination chemotherapy, where it exerts a significant synergistic effect.

Isorhamnetin is a representative flavonoid compound that exhibits antitumor activity primarily by inhibiting tumor cell proliferation, inducing apoptosis, and inhibiting signal transduction. For example, Cai et al. reported that isorhamnetin inhibits the proliferation and metastasis of androgen-independent prostate cancer cells by targeting the endogenous apoptotic pathway and selectively inhibiting $\mathrm{PISK} / \mathrm{AKT} / \mathrm{mTOR}$ signaling [40].

Natural products are highly complex, and their effects on the treatment of diseases may result from the combined action of multiple compounds. Our results showed that quercetin, evodiamine, and isorhamnetin were associated with many NPC targets. GO enrichment analysis showed that NPC targets of Evodia compounds were involved in biological processes related to NPC development, including drug response, generation of ROS, and cellular response to chemical stress. Furthermore, Arij et al. reported that dysfunctional gene-driven redox stress pathways may increase the risk of disease in NPC-susceptible individuals [41].

Uncontrolled tumor cell proliferation is related to abnormal gene expression and signal transduction. The current findings suggested that Evodia may interfere with these pathological processes to inhibit NPC cell proliferation and promote their apoptosis. Our KEGG pathway analysis showed that Evodia compounds affect NPC via multiple signaling pathways that overlap with lipid- and atherosclerosis-related signaling pathways. Analysis of these signaling pathways revealed 37 common biomarkers, including lectin-like oxidized low-density lipoprotein receptor-1 (LOX-1), AKT, nuclear factor (NF)-kB, and p53, which are important in cell proliferation, apoptosis, migration, and invasion. A previous study showed that LOX-1 alters ROS production, leading to lipid peroxidation and DNA damage, and promotes atherosclerosis and cancer progression, suggesting that LOX-1 inhibition may be a promising therapeutic strategy [42]. Therefore, these biomarkers could represent a potential link between atherosclerosis and cancer and provide insight into the associated molecular mechanisms. 
The generated PPI network identified six targets for molecular docking studies, namely MAPK14, AKT1, RELA, JUN, MAPK1, and p53. AKT1 is a key regulator of PI3K/AKT signaling and participates in cell proliferation and apoptosis [43]. MAPK14 and MAPK1 are important members of the MAPK family, and MAPK14 is an important biomarker of metastatic gastric cancer, where its inhibition significantly reduces the progression of advanced gastric cancer [44]. Additionally, MAPK1 affects the progression of breast and ovarian cancers by participating in multiple signaling pathways $[45,46]$, and AKT1 is closely related to cancer and a variety of diseases through its roles in metabolic regulation and multiple signaling pathways [47,48]. RELA is an important target gene in NF-KB signaling; Lu et al. [49] reported that RELA dephosphorylation inhibits tumor development [49], and other studies have indicated that RELA methylation and subsequent activation of multiple downstream genes contribute to the regulation of breast cancer progression [50,51]. JUN is an activator protein- 1 transcription factor subunit that regulates the expression of multiple genes critical to cell proliferation, differentiation, and apoptosis [52], and TP53 is the most commonly mutated tumor suppressor gene in human cancers, with its mutations closely related to a variety of cancers and targeted for the development of numerous treatment strategies [53].

\section{Conclusion}

As a TCM, Evodia rutaecarpa has achieved remarkable results in clinical practice and is an important component in the combination therapy of NPC with other drugs. Reports on the mechanisms of action of natural plants in the treatment of NPC are scarce; therefore, this study offers new insights for the study of Evodia and its applications as adjuvant therapy. Elucidation of the mechanisms through which Evodia rutaecarpa exerts curative effects will be beneficial. In this study, we identified the effective active components of Evodia and target molecules in NPC. Our findings suggested that continued evaluation of evodiamine and other active components is warranted for their possible application in treating NPC and for supporting the scientific basis of using natural plants to treat NPC.

\section{Declarations}

\section{Ethics declarations}

The study was carried out in accordance with the Helsinki Declaration. The databases used in this study are public databases. The study designs involving the respective patients in the databases included obtaining ethical approval, and consent was obtained from all patients and/or their legal guardian(s). Users can download relevant data for free for research and publish relevant articles. The raw data used in this study did not require any administrative authority. The current study was based on open-source data; therefore, it did not require ethical approval. The data used in this study were anonymized before use.

\section{Data availability}

The dataset used in this paper are available from the corresponding author upon request. 


\section{Conflicts of interest}

The authors declared that they have no conflicts of interest regarding this work.

\section{Author contributions}

RSX and YCH designed the study; RSX analyzed and interpreted the data; XMY, YYT, and WL performed the experiments; RSX wrote the manuscript; YX, LH, and FLZ edited and revised the manuscript; and all authors read and approved the final version of the manuscript.

\section{Funding statement}

This work was supported by the National Natural Science Foundation of China [grant nos. 81874408, 82104941]; the Project of Hunan Provincial Department of Education [grant nos. 21A0229, 21B0359]; the Project of Hunan Provincial Health Commission [grant no. 202207015643]; the College Student Innovation Project of Hunan Province [grant no. S202010541029]; and the Domestic First-class Discipline Construction Project of Chinese Medicine of Hunan University of Chinese Medicine[grant no.[2018]469].

\section{Acknowledgments}

The authors would like to thank the TCMSP, Genecards, OMIM, Drugbank, TTD, PharmGkb, STRING, RCSB PDB, TCGA, and HPA databases for the availability of data.The study has been announced in Research Square Preprint Platform.

\section{References}

[1] H. Sung, J. Ferlay, R. L. Siegel et al., "Global cancer Statistics 2020: GLOBOCAN estimates of incidence and mortality worldwide for 36 cancers in 185 countries," CA: A Cancer Journal for Clinicians, vol. 71, pp. 209-249, 2021.

[2] I. Argirion, K. R. Zarins, K. Suwanrungruang et al., "Subtype specific nasopharyngeal carcinoma incidence and survival trends: differences between endemic and non-endemic populations," Asian Pacific Journal of Cancer Prevention, vol. 21, pp. 3291-3299, 2020.

[3] Y. Zhang, L. Chen, G. Q. Hu et al., "Gemcitabine and cisplatin induction chemotherapy in nasopharyngeal carcinoma," New England Journal of Medicine, vol. 381, pp. 1124-1135, 2019.

[4] S. Poirier, G. Bouvier, C. Malaveille et al., "Volatile nitrosamine levels and genotoxicity of food samples from high-risk areas for nasopharyngeal carcinoma before and after nitrosation," International Journal of Cancer, vol. 44, pp. 1088-1094, 1989.

[5] C. Shannon-Lowe and A. Rickinson, "The global landscape of EBV-associated tumors," Frontiers in Oncology, vol. 9, pp. 713, 2019. 
[6] A. T. Chan, V. Grégoire, J. L. Lefebvre et al., "EHNS-ESMO-ESTRO Guidelines Working Group, Nasopharyngeal cancer: EHNS-ESMO-ESTRO Clinical Practice Guidelines for diagnosis, treatment and follow-up," Annals of Oncology, vol. 23, pp. vii83-vii85, 2012.

[7] J. Jiang and C. Hu, "Evodiamine: a novel anti-cancer alkaloid from Evodia rutaecarpa," Molecules, vol. 14, pp. 1852-1859, 2009.

[8] D. W. Li, M. Zhang, L. Feng et al., "Alkaloids from the nearly ripe fruits of Evodia rutaecarpa and their bioactivities," Fitoterapia, vol. 146, pp. 104668, 2020.

[9] S. S. Liu, Y. T. Dai, F. Sui et al., "Flavonol glycosides from the fruits of Evodia rutaecarpa," Journal of Asian Natural Products Research, vol. 20, pp. 867-874, 2020.

[10] K. M. Tian, J. J. Li, and S. W. Xu, "Rutaecarpine: a promising cardiovascular protective alkaloid from Evodia rutaecarpa (Wu Zhu Yu)," Pharmacology Research, vol. 141, pp. 541-550, 2019.

[11] J. Ru, P. Li, J. Wang et al., "TCMSP: a database of systems pharmacology for drug discovery from herbal medicines," Journal of Cheminformatics, vol. 6, pp. 13, 2014.

[12] X. Xu, W. Zhang, C. Huang, et al., "A novel chemometric method for the prediction of human oral bioavailability," International Journal of Molecular Science, vol. 13, pp. 6964-6982, 2012.

[13] L. Breuza, S. Poux, A. Estreicher et al., "UniProt, The UniProtKB guide to the human proteome," Database, Oxford, 2016.

[14] P. Shannon, A. Markiel, O. Ozier et al., "Cytoscape: a software environment for integrated models of biomolecular interaction networks," Genome Research, vol. 13, pp. 2498-2504, 2003.

[15] A. L. Hopkins, "Network pharmacology: the next paradigm in drug discovery," Nature Chemical Biology, vol. 4, pp. 682-690, 2008.

[16] H. Yu, P. M. Kim, E. Sprecher, V. Trifonov, and M. Gerstein, "The importance of bottlenecks in protein networks: correlation with gene essentiality and expression dynamics," PLOS Computational Biology, vol. 3, pp. e59, 2007.

[17] A. Athanasios, V. Charalampos, T. Vasileios, and G. M. Ashraf, "Protein-protein interaction (PPI) network: recent advances in drug discovery," Current Drug Metabolism, vol. 18, pp. 5-10, 2017.

[18] Y. Tang, M. Li, J. Wang, Y. Pan, and F. X. Wu, "CytoNCA: a cytoscape plugin for centrality analysis and evaluation of protein interaction network," Biosystems, vol. 127, pp. 67-72, 2015.

[19] Gene Ontology Consortium, "Gene Ontology Consortium: going forward," Nucleic Acids Research, vol. 43, pp. D1049-D1056, 2015. 
[20] M. Kanehisa and S. Goto, "KEGG: Kyoto Encyclopedia of Genes and Genomes," Nucleic Acids Research, vol. 28, pp. 27-30, 2000.

[21] G. Yu, L. G. Wang, Y. Han, and Q. Y. He, "clusterProfiler: an R package for comparing biological themes among gene clusters," Omics, vol. 16, pp. 284-287, 2012.

[22] S. Kim, B. A. Shoemaker, E. E. Bolton, and S. H. Bryant, “Finding potential multitarget ligands using PubChem," Methods in Molecular Biology, vol. 1825, pp. 63-91, 2018.

[23] 0. Trott, and A. J. Olson, "AutoDock Vina: improving the speed and accuracy of docking with a new scoring function, efficient optimization, and multithreading," Journal of Computational Chemistry, vol. 31, pp. 455-461, 2010.

[24] P. J. Thul, and C. Lindskog, "The human protein atlas: a spatial map of the human proteome," Protein Science, vol. 27, pp. 233-244, 2018.

[25] F. Zhou, J. Hu, N. Dai et al., "Berberine and ginsenoside Rg3 act synergistically via the MAPK/ERK pathway in nasopharyngeal carcinoma cells," Journal of Functional Foods, vol. 66, pp. 103802, 2020.

[26] M. Reyes-Farias and C. Carrasco-Pozo, "The anti-cancer effect of quercetin: molecular implications in cancer metabolism," International Journal of Molecular Science, vol. 20, 2019.

[27] Y. P. Chen, A. T. C. Chan, Q. T. Le, P. Blanchard, Y. Sun, and J. Ma, "Nasopharyngeal carcinoma," Lancet, vol. 394, pp. 64-80, 2019.

[28] H. Matsuda, J. X. Wu, T. Tanaka, M. linuma, and M. Kubo, "Antinociceptive activities of $70 \%$ methanol extract of evodiae fructus (fruit of Evodia rutaecarpa var. bodinieri) and its alkaloidal components," Biological and Pharmaceutical Bulletin, vol. 20, pp. 243-248, 1997.

[29] T. C. Moon, M. Murakami, I. Kudo et al., "A new class of COX-2 inhibitor, rutaecarpine from Evodia rutaecarpa," Inflammation Research, vol. 48, pp. 621-625, 1999.

[30] T. C. Rho, E. A. Bae, D. H. Kim et al., "Anti-Helicobacter pylori activity of quinolone alkaloids from Evodiae fructus," Biological and Pharmaceutical Bulletin, vol. 22, pp. 1141-1143, 1999.

[31] C. H. Liao, S. L. Pan, J. H. Guh et al., "Antitumor mechanism of evodiamine, a constituent from Chinese herb Evodiae fructus, in human multiple-drug resistant breast cancer NCI/ADR-RES cells in vitro and in vivo," Carcinogenesis, vol. 26, pp. 968-975, 2005.

[32] J. Y. Hong, S. H. Park, H. Y. Min, H. J. Park, and S. K. Lee, "Anti-proliferative effects of evodiamine in human lung cancer cells," Journal of Cancer Prevention, vol. 19, pp. 7-13, 2014.

[33] J. R. Bae, W. H. Park, D. H. Suh, Y. B. Kim, and K. Kim, "Role of limonin in anticancer effects of Evodia rutaecarpa on ovarian cancer cells," BMC Complementary Medicine and Therapies, vol. 20, pp. 94, 2020. 
[34] T. C. Chen, C. C. Chien, M. S. Wu, and Y. C. Chen, "Evodiamine from Evodia rutaecarpa induces apoptosis via activation of JNK and PERK in human ovarian cancer cells," Phytomedicine, vol. 23, pp. 68-78, 2016.

[35] C. Y. Hu, H. T. Wu, Y. C. Su, C. H. Lin, C. J. Chang, and C. L. Wu, “Evodiamine exerts an antihepatocellular carcinoma activity through a WWOX-dependent pathway," Molecules, vol. 22, 2017.

[36] T. Zhang, S. Qu, Q. Shi, D. He, and X. Jin, “Evodiamine induces apoptosis and enhances TRAILinduced apoptosis in human bladder cancer cells through mTOR/S6K1-mediated downregulation of Mcl1," International Journal of Molecular Science, vol. 15, pp. 3154-3171, 2014.

[37] D. Y. Huang, Z. R. Dai, W. M. Li, R. G. Wang, and S. M. Yang, "Inhibition of EGF expression and NF-kB activity by treatment with quercetin leads to suppression of angiogenesis in nasopharyngeal carcinoma," Saudi Journal of Biological Science, vol. 25, pp. 826-831, 2018.

[38] M. Daker, M. Ahmad, and A. S. Khoo, "Quercetin-induced inhibition and synergistic activity with cisplatin - a chemotherapeutic strategy for nasopharyngeal carcinoma cells," Cancer Cell International, vol. 12, pp. 34, 2012.

[39] F. Zhang, Y. Cui, and P. Cao, "Effect of quercetin on proliferation and apoptosis of human nasopharyngeal carcinoma HEN1 cells," Journal of Huazhong University of Science and Technology. Medical Sciences, vol. 28, pp. 369-372, 2008.

[40] F. Cai, Y. Zhang, J. Li, S. Huang, and R. Gao, "Isorhamnetin inhibited the proliferation and metastasis of androgen-independent prostate cancer cells by targeting the mitochondrion-dependent intrinsic apoptotic and PI3K/Akt/mTOR pathway," Bioscience Reports, vol. 40, 2020.

[41] A. B. Chaaben, C. Mariaselvam, S. Salah et al., "Polymorphisms in oxidative stress-related genes are associated with nasopharyngeal carcinoma susceptibility," Immunobiology, vol. 220, pp. 20-25, 2015.

[42] S. Balzan, and V. Lubrano, "LOX-1 receptor: a potential link in atherosclerosis and cancer," Life Sciences, vol. 198, p. 79-86, 2018.

[43] N. Balasuriya, M. McKenna, X. Liu, S. S. C. Li, and P. O’Donoghue, "Phosphorylation-dependent inhibition of Akt1," Genes (Basel), vol. 92018, 2018.

[44] F. P. Mesquita, C. A. Moreira-Nunes, E. L. da Silva et al., "MAPK14 (p38a) inhibition effects against metastatic gastric cancer cells: a potential biomarker and pharmacological target," Toxicology In Vitro, vol. 66, pp. 104839, 2020.

[45] R. Deng, H. L. Zhang, J. H. Huang et al. "MAPK1/3 kinase-dependent ULK1 degradation attenuates mitophagy and promotes breast cancer bone metastasis," Autophagy, vol. 17, pp. 3011-3029, 2021. 
[46] T. T. Yu, C. Y. Wang, and R. Tong, “ERBB2 gene expression silencing involved in ovarian cancer cell migration and invasion through mediating MAPK1/MAPK3 signaling pathway," European Review for Medical and Pharmacological Sciences, vol. 24, pp. 5267-5280, 2020.

[47] D. Massihnia, A. Avan, N. Funel et al., "Phospho-Akt overexpression is prognostic and can be used to tailor the synergistic interaction of Akt inhibitors with gemcitabine in pancreatic cancer," Journal of Hematology and Oncology, vol. 10, pp. 9, 2017.

[48] A. Slipicevic, R. Holm, M. T. Nguyen, P. J. Bøhler, B. Davidson, and V. A. Flørenes, “Expression of activated Akt and PTEN in malignant melanomas: relationship with clinical outcome," American Journal of Clinical Pathology, vol. 124, pp. 528-536, 2005.

[49] X. Lu, and W. G. Yarbrough, "Negative regulation of RelA phosphorylation: emerging players and their roles in cancer," Cytokine \& Growth Factor Reviews, vol. 26, pp. 7-13, 2015.

[50] Y. J. Jeong, H. K. Oh, and H. R. Choi, "Methylation of the RELA gene is associated with expression of NF-kappaB1 in response to TNF-alpha in breast," Journal of Cancer Molecules, vol. 24, 2019.

[51] S. Biswas, S. R. Chowdhury, G. Mandal, S. Purohit, A. Gupta, and A. Bhattacharyya, "RelA driven coexpression of CXCL13 and CXCR5 is governed by a multifaceted transcriptional program regulating breast cancer progression," Biochimica et Biophysicia Acta Molecular Basis of Disease, vol. 1865, pp. 502-511, 2019.

[52] M. Hartl, A. G. Bader, and K. Bister, "Molecular targets of the oncogenic transcription factor jun," Current Cancer Drug Targets, vol. 3, pp. 41-55, 2003.

[53] G. Zhu, C. Pan, J. X. Bei, et al., "Mutant p53 in cancer progression and targeted therapies," Frontiers in Oncology, vol. 10, pp. 595187, 2020.

\section{Abbreviations}

Nasopharyngeal carcinoma (NPC)

Gene Ontology (GO)

Kyoto Encyclopedia of Genes and Genomes (KEGG)

The Cancer Genome Atlas (TCGA)

Human Protein Atlas (HPA)

Epstein-Barr virus (EBV)

Traditional Chinese medicine (TCM) 
TCM Systems Pharmacology (TCMSP)

Oral bioavailability (OB)

Drug-likeness (DL)

Betweenness centrality (BC)

Closeness centrality (CC)

Protein-protein interaction (PPI)

Degree centrality (DC),

Eigenvector centrality (EC)

Network centrality (NC)

Local average connectivity (LAC)

Figures 
Date Preparation

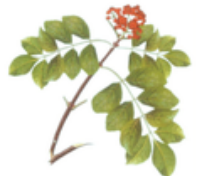

Evodia Rutaecarpa

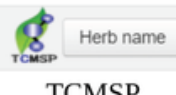

TCMSP

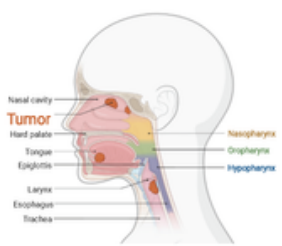

Nasopharyngeal Carcinoma

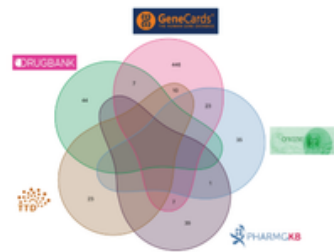

GeneCards/OMIM/ PharmGKB/TTD/DrugBank
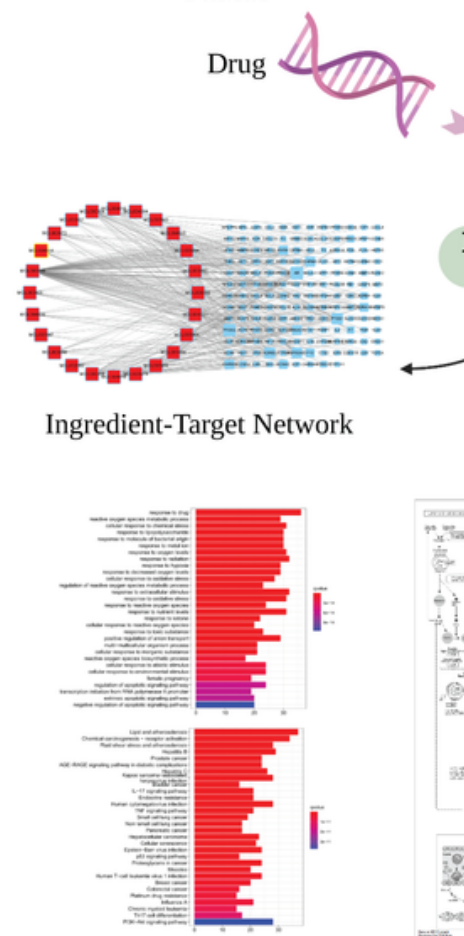

GO and KRGG Enrichment Enrichment Analysis
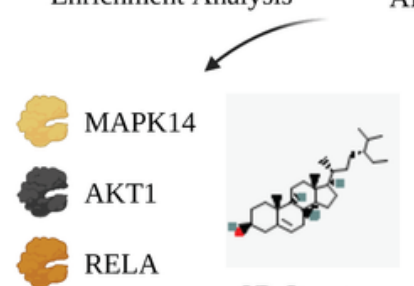
2D Structure
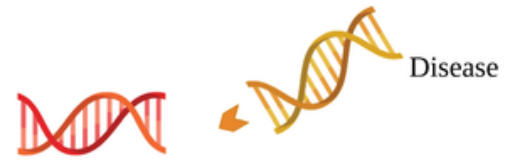

Common Gene
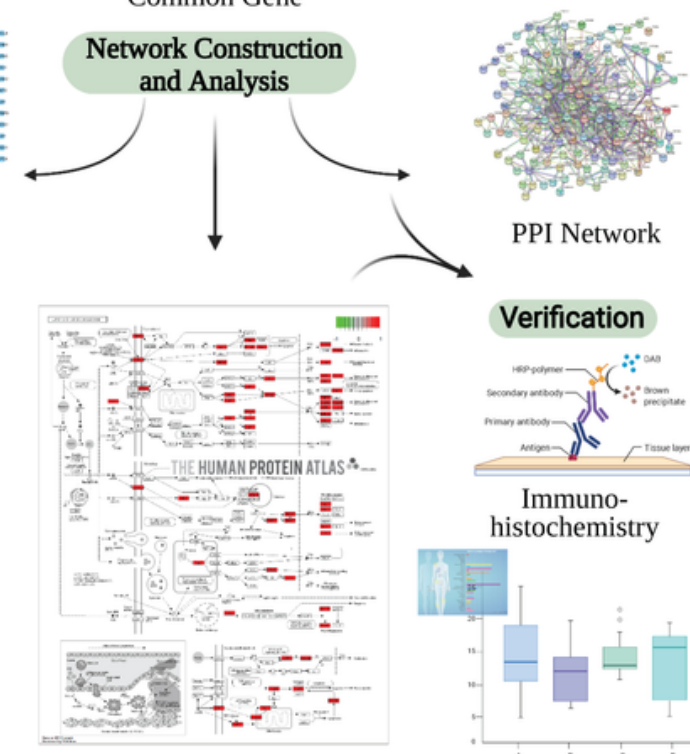

Potential Targets on LIPID AND ATHEROSCLERSOSIS

\section{Molecular Docking}

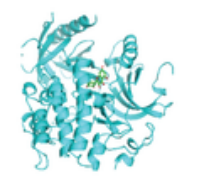

3D Molecular Docking Diagrams

Verification
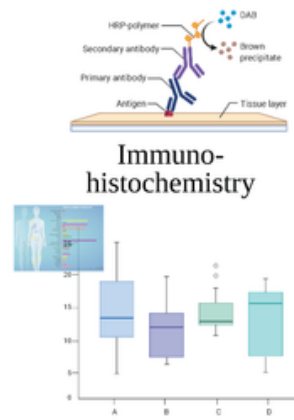

Differential

Expression Analysis

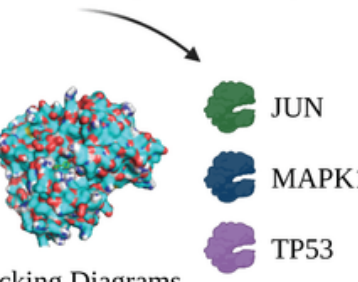$$
\checkmark
$$

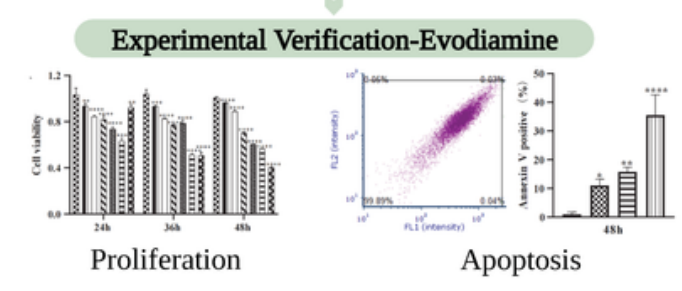

\section{Figure 1}

Flow diagram of the systematic analysis and validation processes. 
A

B

DrugBank

Genecards
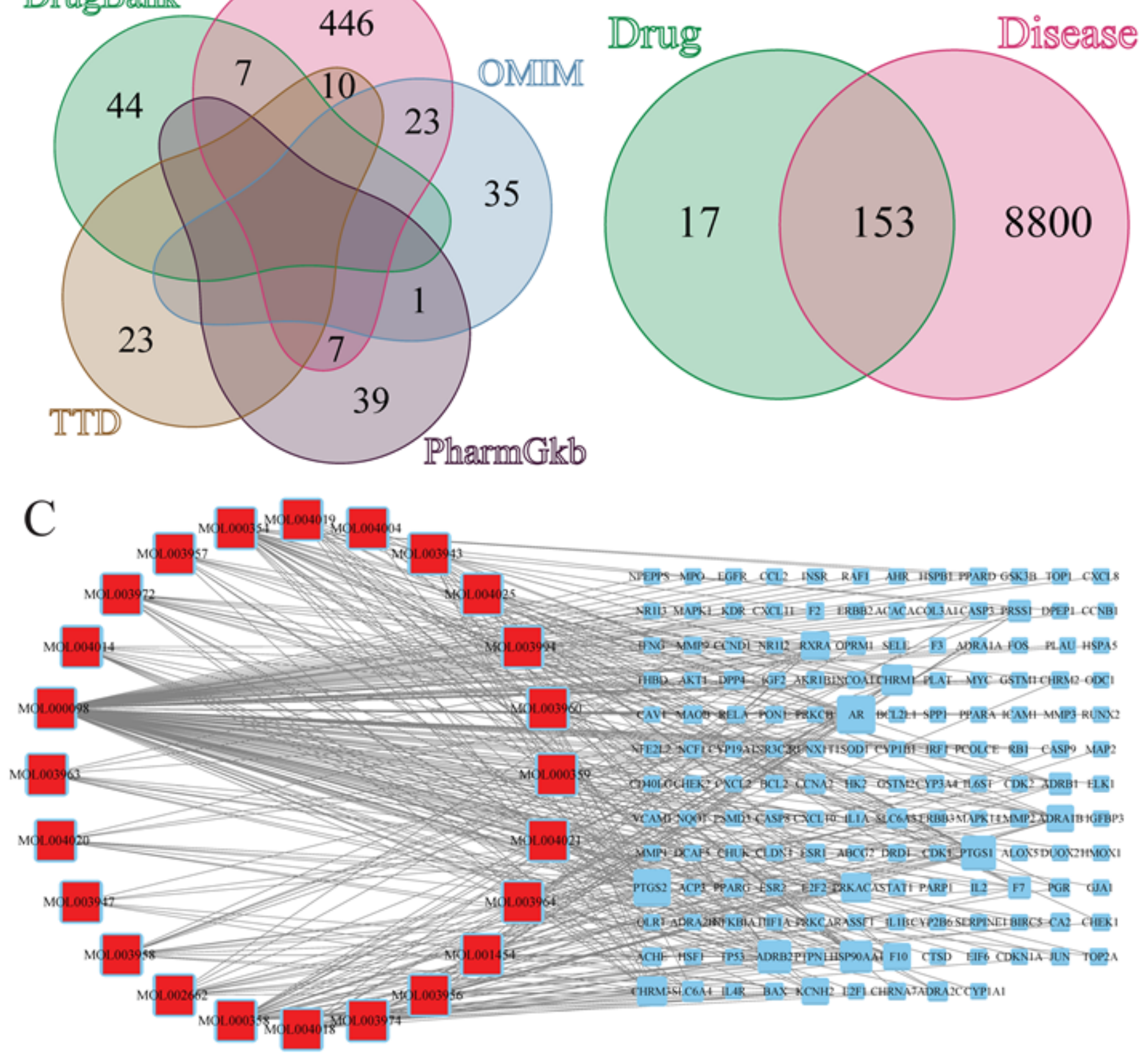

Figure 2

Construction of the target gene set and compound-target network. (A) Venn diagram of NPC target genes from the GeneCard, OMIM, PharmGkb, TTD, and Drugbank databases. (B) Venn diagram of Evodia compounds and NPC targets. (C) Evodia compound-target network. The red rectangular nodes are the main active Evodia compounds, and the blue rectangle is the potential NPC target for treatment using the Evodia compounds. 

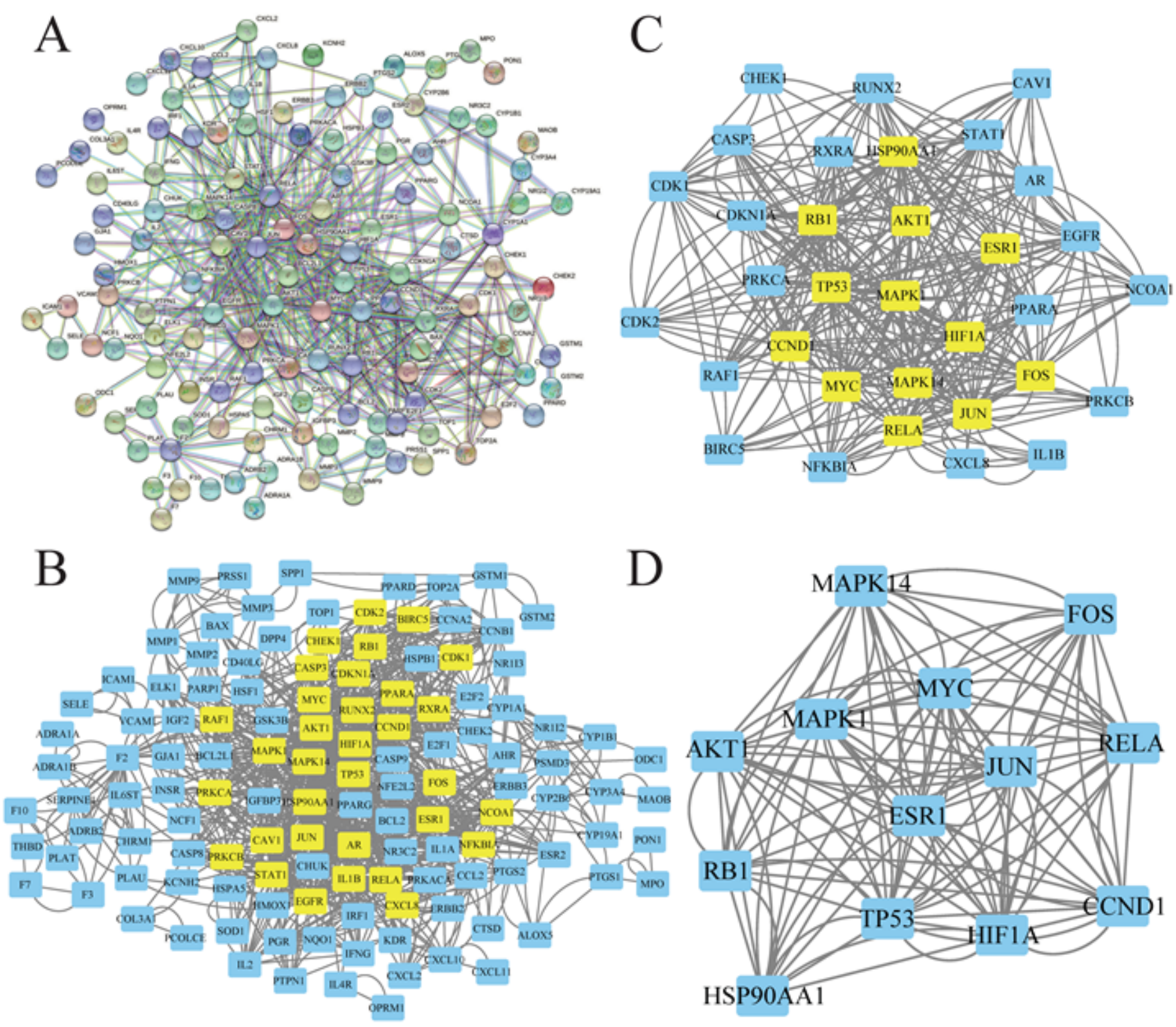

Figure 3

Generated PPI network. (A) PPI network of NPC targets of Evodia compounds. (B) PPI network containing 127 nodes and 1006 edges. Areas highlighted in yellow represent the candidate targets ranked according to median values of DC, BC, CC, EC, LAC, and NC. (C) PPI network containing 33 nodes and 363 edges. Areas highlighted in yellow represent potential targets with median values of DC, BC, CC, EC, LAC, and NC. (D) PPI network containing 13 potential targets with median values for DC, BC, CC, EC, LAC, and NC. 


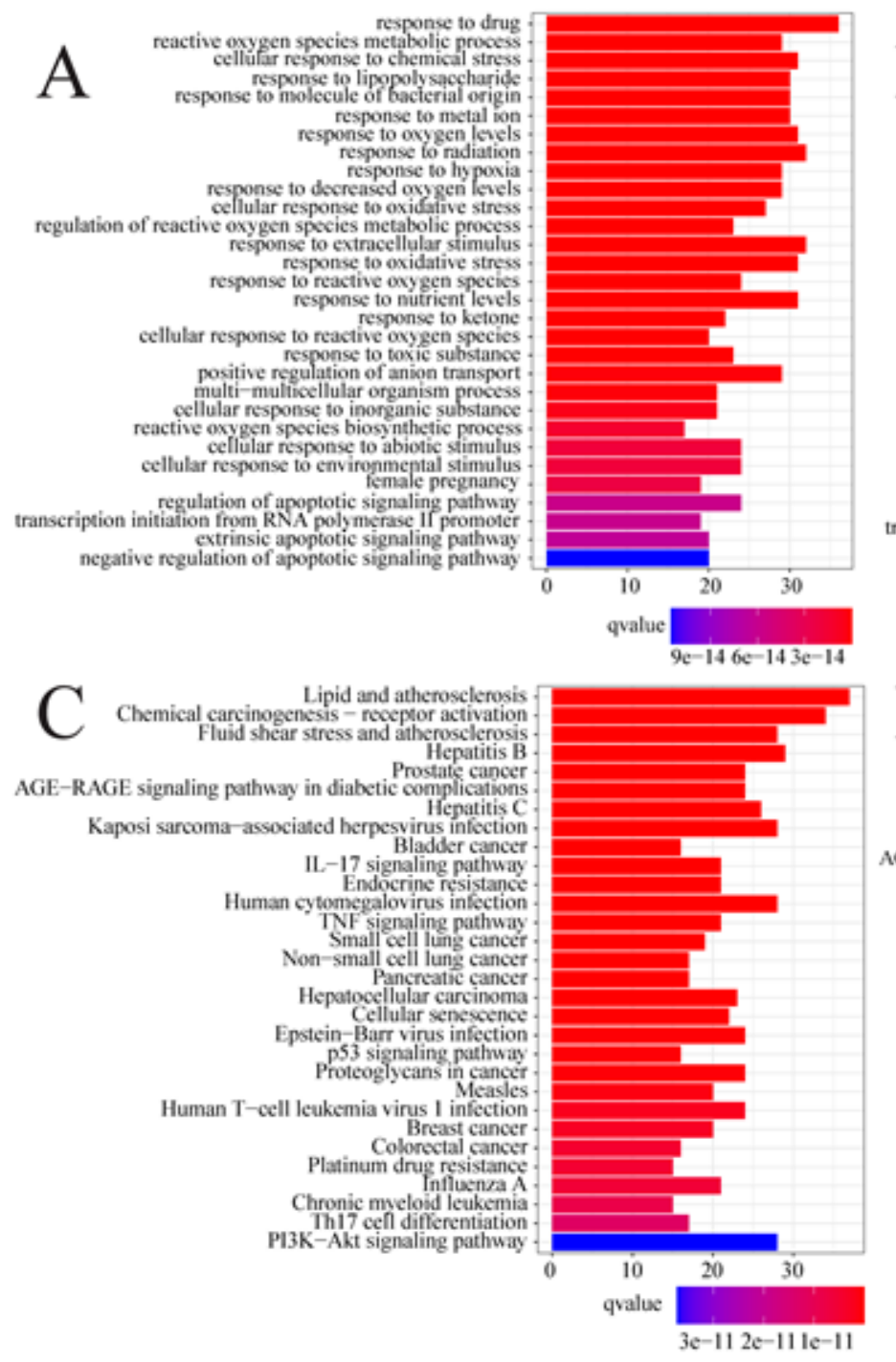

B
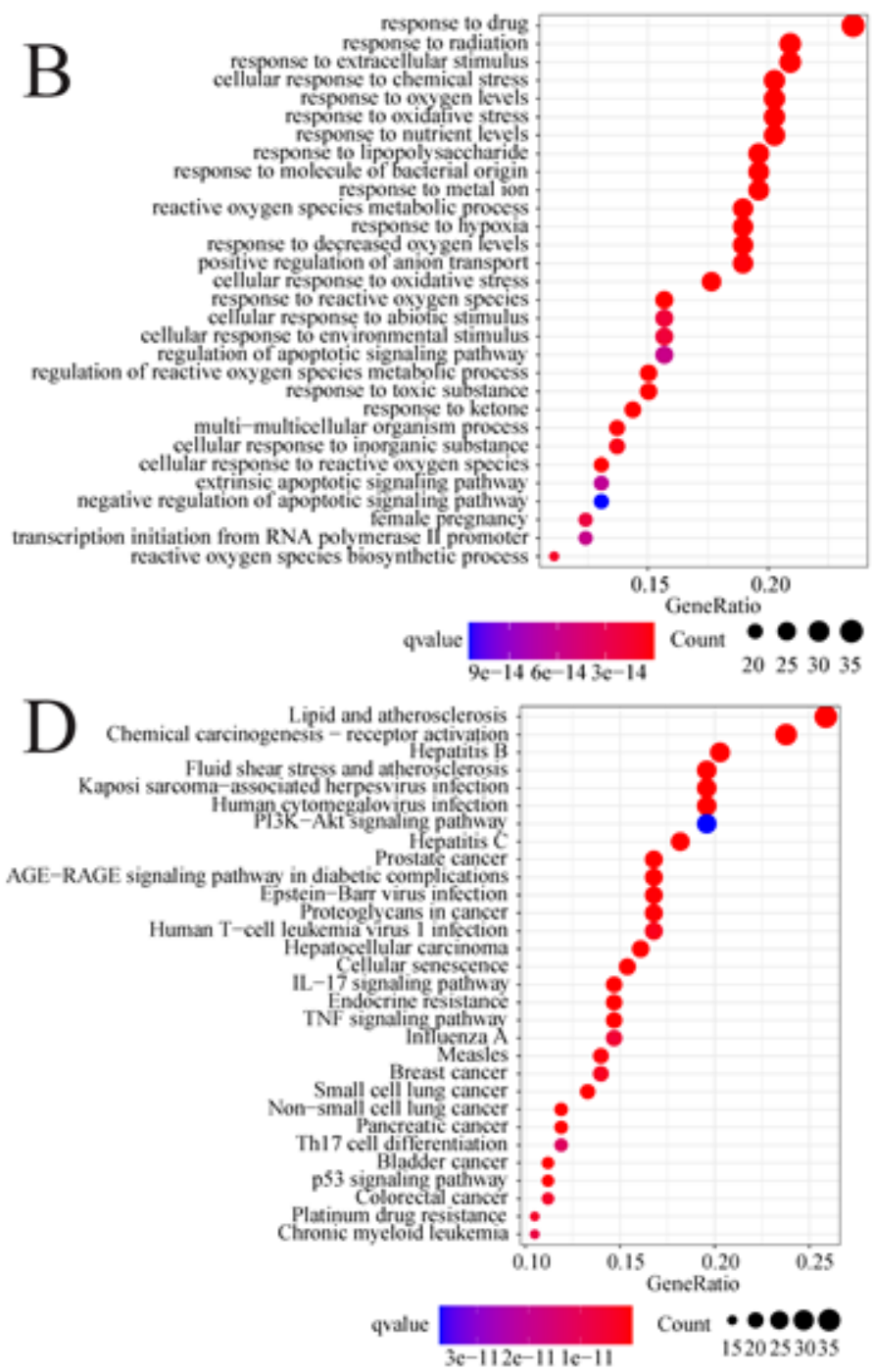

Figure 4

Top 20 GO enriched terms and KEGG pathway annotations. (A, B) GO enrichment of active Evodia compounds for application against NPC targets. (C, D) Enriched KEGG pathways of potential NPC targets using active Evodia compounds. 


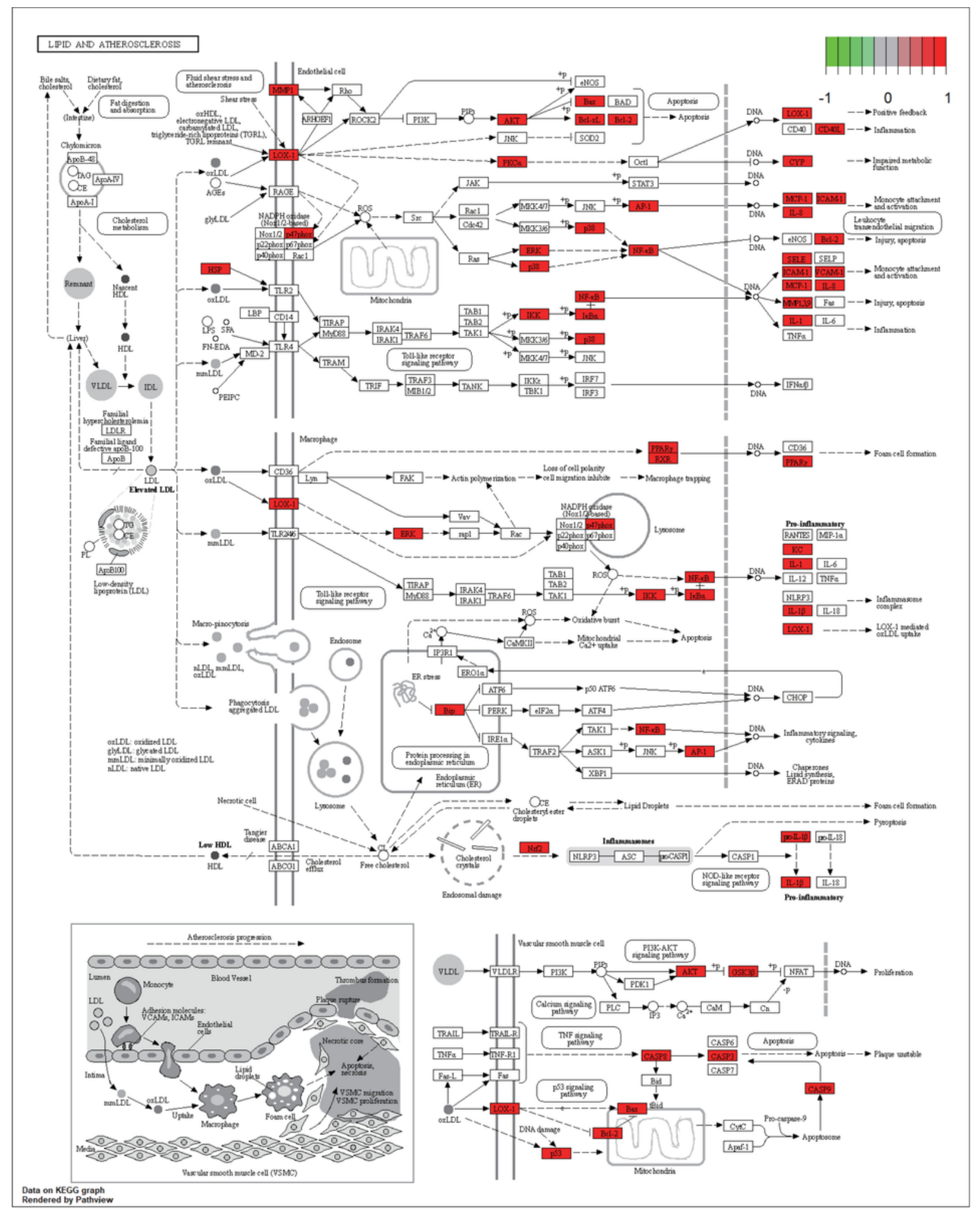

\section{Figure 5}

Pathway map of Evodia compounds for their potential treatment of NPC. NPC targets related to the Evodia compounds are involved in "lipid and atherosclerosis" pathways. Arrows represent activation, Tarrows represent inhibition, and segments show either activation or inhibition. 


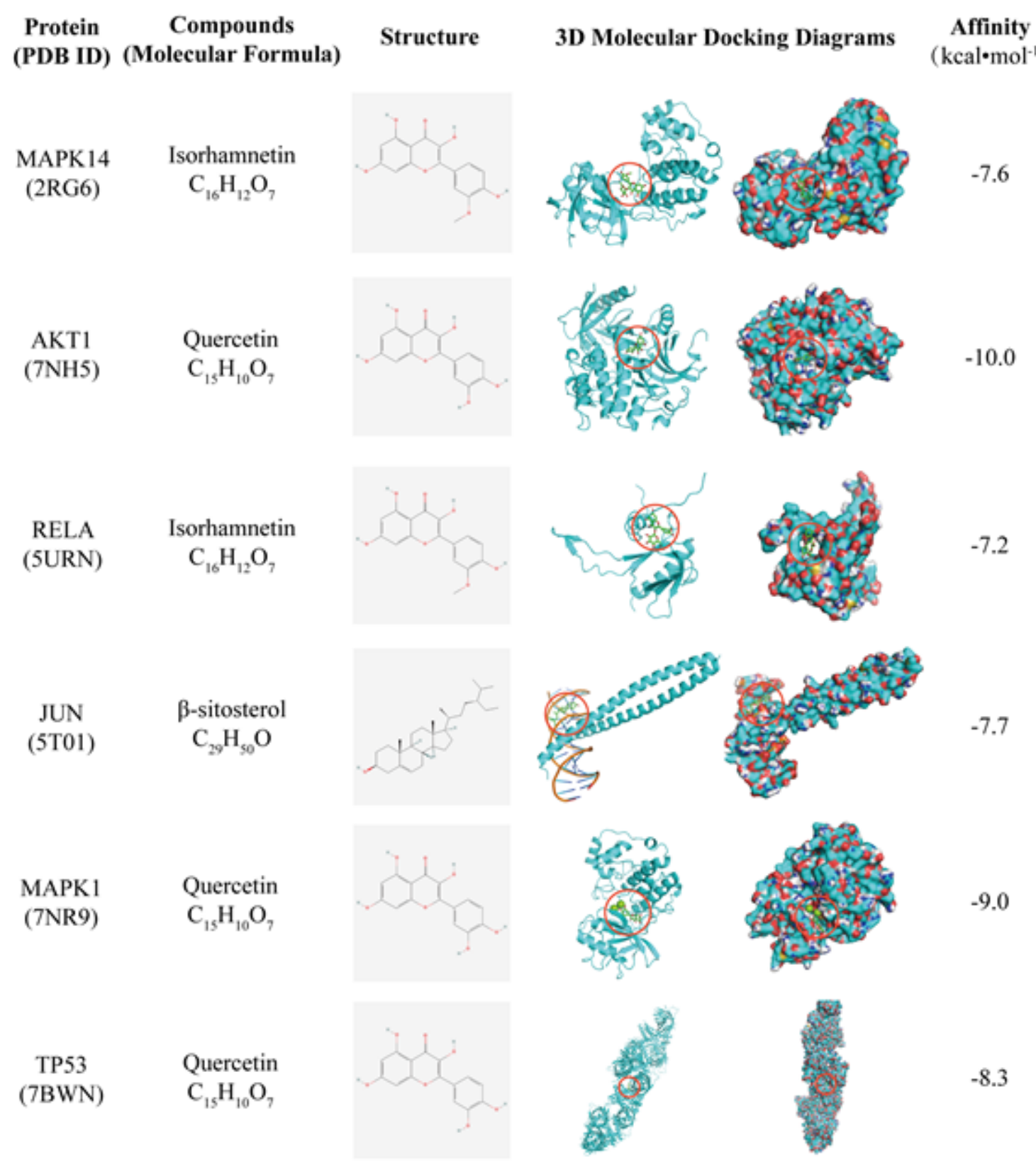

\section{Figure 6}

Molecular docking of Evodia compounds with NPC targets. 

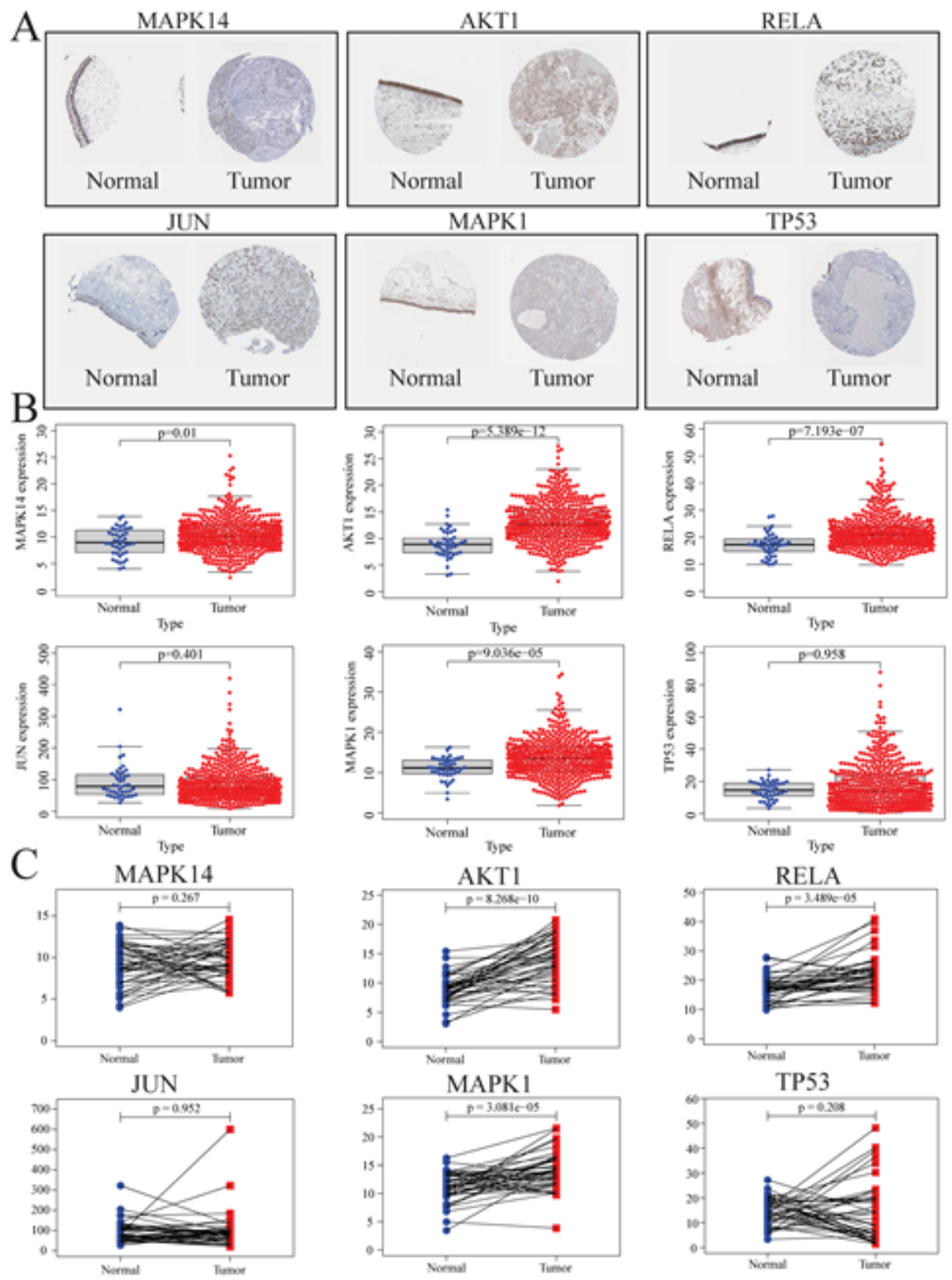

\section{Figure 7}

Differential expression of NPC targets. (A) Validation of the expression of NPC targets at the protein level using the HPA database (immunohistochemistry). (B) Differential expression of NPC targets. (C) Paired difference analysis of NPC targets. 


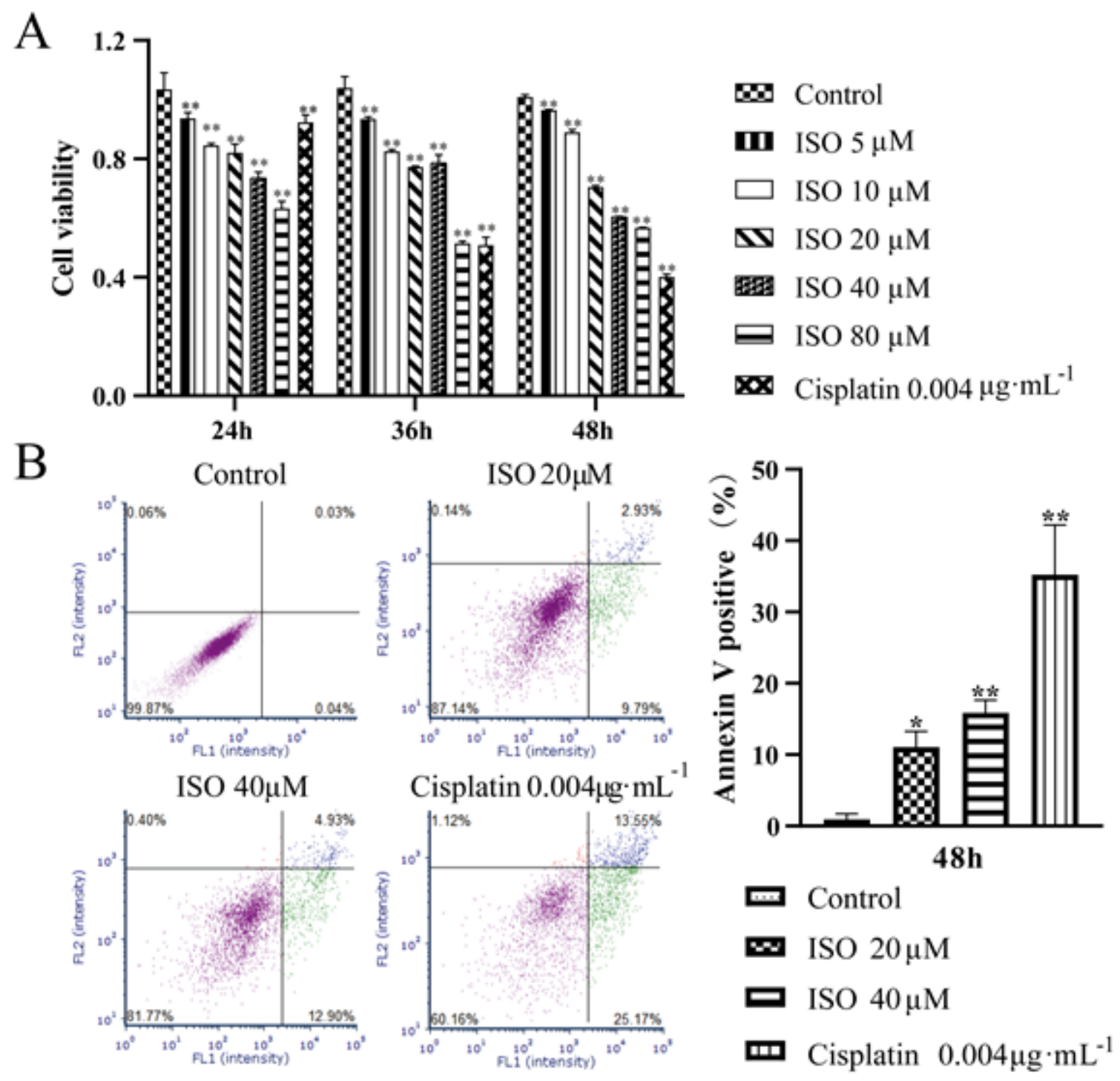

Lower Left: Live $\square$ Lower Right:Apoptotic $\square$ Upper Right:Necrotic $\square$ Upper Left: Debris

Figure 8 Experimental verification. (A) Isorhamnetin inhibits the proliferation of CNE2 cells according to CCK-8 assay $(\overline{\mathrm{x}} \pm s, n=3)$. (B) Isorhamnetin promotes CNE2 cell apoptosis $(\mathrm{x} \pm \mathrm{s}, \mathrm{n}=3)$.

Figure 8

See figure for legend.

\section{Supplementary Files}

This is a list of supplementary files associated with this preprint. Click to download.

- SupplementaryTables.xlsx 